\title{
Abandono del hábito tabáquico tras un primer ingreso en el Servicio de Neumología
}

\author{
EVA CABRERA C.*, MARÍA C. FERNÁNDEZ A.*, JOSÉ L. VELASCO G.*, NURIA REINA M.*, \\ LIDIA LÓPEZ L.*, MARÍA C. VERA S.*, LORENA PIÑEL J.* y MARÍA V. HIDALGO S.*
}

\section{Smoking cessation after first admission in a respiratory unit}

Some authors suggest that hospitalization is an ideal moment to stop smoking. It is unknown the outcome of smokers admitted for the first time in the pulmonology department, which interventions are carried out to achieve smoking cessation and which are the dropout rates once they are discharged. The purpose of this study is to analyze the impact of a first hospital admission in a service of Pneumology on smokers. Epidemiological variables, smoking habit, specialized interventions carried out on these subjects and rate of withdrawal after discharge were studied. It is necessary to train specialists to diagnose and treat the patient in the process, so that on every hospitalized smoker a cessation intervention should be carried out.

Key words: Smoking, hospital admission, smoking cessation rates, smokers, withdrawal, medical advice.

\section{Resumen}

Algunos autores defienden que el ingreso hospitalario constituye un marco ideal para dejar de fumar. Se desconoce lo que sucede con los fumadores que ingresan por primera vez en el Servicio de Neumología, sobre las intervenciones que en ellos se lleva a cabo, así como sus tasas de abandono una vez que son dados de alta. El objetivo de este estudio es analizar el impacto que tiene un primer ingreso hospitalario en el Servicio de Neumología en sujetos fumadores. Conocer sus variables epidemiológicas, el hábito de tabáquico, las intervenciones que el personal médico especializado realiza sobre estos sujetos y las tasas de abstinencia posteriores al alta. Es necesario formar a los especialistas para diagnosticar y tratar al paciente en dicho proceso, de tal forma que sobre todo sujeto fumador hospitalizado se realice alguna intervención.

Palabras clave: Tabaquismo, ingreso hospitalario, tasa de abandono, fumadores, abstinencia, intervención.

\section{Introducción}

El tabaco es la primera causa de morbimortalidad prevenible, constituyendo unos de los principales problemas de salud a nivel mundial. En España en el año 2006 más de cincuenta mil defunciones se relacionaron con su consumo, lo que supone que una de cada siete muertes ocurridas en individuos mayores de 35 años es atribuible al tabaco ${ }^{1}$. Es causa directa de diferentes tipos de neoplasias ${ }^{2}$, de enfermedades cardiovasculares ${ }^{3}$ y de patologías respiratorias, entre otras ${ }^{4}$, de ahí la necesidad de una educación social e iniciativas que disminuyan su consumo como las reguladas en el Convenio Marco del Control del Tabaco que define una normativa para abordar las cuestiones relativas al consumo de tabaco, afirmando la importancia de la reducción de la demanda, así como de ciertas cuestiones relativas al suministro. Algunos autores defienden que el ingreso hospitalario constituye un marco ideal para dejar de fumar ${ }^{5,6}$, se dan diferentes facto-

* Hospital Universitario Virgen de la Victoria, Málaga, España. 
res que pueden ayudar al abandono del tabaco: el ser un espacio libre de humo, la asociación de los síntomas que provocan el ingreso con el consumo del tabaco y la presencia del personal sanitario. En los pacientes con patología cardiovascular se han realizado diferentes estudios que relacionan sus tasas de abandono con distintas intervenciones y la abstinencia registrada una vez que son dados de alta. Sin embargo, se ha publicado menos sobre los fumadores con patología pulmonar, sobre las intervenciones que en ellos se lleva a cabo, así como sus tasas de abandono una vez que son dados de alta. El objetivo de este estudio es analizar el impacto que tiene un primer ingreso hospitalario en el Servicio de Neumología en sujetos fumadores. Conocer sus variables epidemiológicas, el hábito tabáquico, las intervenciones que el personal médico especializado realiza sobre estos sujetos y las tasas de abstinencia posteriores al alta. Además intentar establecer una relación entre el diagnóstico y la tasa de abandono del tabaco.

\section{Metodología}

Se ha realizado un trabajo ambispectivo y observacional no intervencionista sobre el hábito tabáquico de los pacientes fumadores ingresados por primera vez en el Servicio de Neumología del Hospital Universitario Virgen de la Victoria de Málaga, España. Se obtuvo la autorización del proyecto por la Comisión de Investigación de este hospital y el Comité ético, certificando que se garantizan todos los aspectos éticos y se salvaguarda la confidencialidad de los pacientes. Se ha realizado según las normas de "Buena Práctica Clínica" y se contemplan los aspectos éticos señalados en la Declaración de Helsinki y las leyes al respecto. El marco muestral utilizado corresponde al censo estadístico del hospital, recogiéndose las historias clínicas de todos los fumadores ingresados por primera vez en el Servicio de Neumología durante el período comprendido entre abril de 2013 y abril de 2014. A través de los informes de alta de dichos sujetos se recogieron variables epidemiológicas, variables clínicas y hábito tabáquico previo al ingreso y tras el mismo, con un cuestionario sencillo (Tabla 1) realizado siempre por el mismo entrevistador a través de llamadas telefónicas. Los sujetos que afirmaban haber abandonado el tabaco, un año después de haber sido dados de alta, acudían al centro para realizar una COoximetría. Los análisis estadísticos fueron realizados con $R$ software versión 3.0.1 (R Foundation for Statistical Computing, Vienna, Australia).

\section{Resultados}

Se revisó un total de 219 historias clínicas de fumadores que ingresaron en el Servicio de Neumología por primera vez en el período comprendido entre abril de 2013 y abril de 2014, de ellos 155 eran hombres $(70,8 \%)$ y 64 mujeres $(29,2 \%)$. La edad media era de 56,3 años ( $\pm 15,5$ DS). Predominaban los sujetos con un nivel de estudios primario $(\mathrm{n}=76 ; 48,1 \%)$. Eran sanos 73 de ellos $(33,3 \%)$, mientras que ya habían sido diagnosticados de patología pulmonar previamente 63 $(29,4 \%), 73$ sujetos $(33,0 \%)$ estaban diagnosticados de hipertensión arterial, siendo diabéticos 38 (18\%), dislipémicos 40 (18,1\%). Estaban siendo tratados por enfermedad psiquiátrica $16(7,2 \%) \mathrm{y}$ $45(20,4 \%)$ contaban con otros diagnósticos.

No presentaban disnea previamente al ingreso el 49,3\% (108 sujetos), se cuantificó por la escala de disnea modificada (mMRC). Referían disnea grado 1, 51 de ellos $(23,3 \%), 30(13,7 \%)$ disnea grado 2 , grado 3 en $22(10,0 \%)$ y $8(3,6 \%)$ tenían disnea grado 4 . La expectoración estaba presente en 101 (46,1\%). Habían estado ingresados en una o dos ocasiones en otros servicios 72 sujetos $(32,9 \%)$ y 13 de ellos $(5,9 \%)$ en más ocasiones. No tenían ninguna estancia hospitalaria previamente $134(61,2 \%)$. Entre los sujetos

Tabla 1. Cuestionario telefónico a los pacientes tras ser dados de alta del Servicio de Neumología

Abandono del tabaco tras un primer ingreso en el Servicio de Neumología

1. ¿Fuma actualmente?

- Sí (1)

- No (2)

2. Si usted sigue fumando:

- ¿Estuvo algún tiempo sin fumar tras el ingreso en Neumología?

- Sí ¿Durante cuánto tiempo? (Semanas) (1. Número de semanas)

- $\quad$ No (2)

3. Si usted ha dejado de fumar: -¿Lo hizo tras el ingreso?

- Sí (1)

- $\quad$ No (2)

- ¿Utilizó algún tipo de terapia?

- Sí ¿Durante cuánto tiempo? (Semanas)

(1. Número de semanas)

- No (2) 
que habían dejado de fumar el 33,3\% de ellos había tenido ingresos previos y en el $66,7 \%$ ésta era la primera vez que ingresaban en el hospital. De los que continúan fumando el $52,6 \%$ era su primer ingreso en el hospital frente a un $47,4 \%$ que habían estado hospitalizados en alguna otra ocasión (Tabla 2).

Tabla 2. Características epidemiológicas de los pacientes ingresados por primera vez en el Servicio de Neumología

\begin{tabular}{|llr|}
\hline Variables & Resultados & \\
\hline Edad promedio (años) & 56,3 & \\
& Hombre & $155(70,8 \%)$ \\
& Mujer & $64(29,2 \%)$ \\
Disnea & 0 & $108(49,8 \%)$ \\
& 1 & $51(23,3 \%)$ \\
& 2 & $30(13,7 \%)$ \\
Expectoración & 3 & $22(10,0 \%)$ \\
& 4 & $8(3,7 \%)$ \\
Comorbilidad & Sí & $101(46,1 \%)$ \\
& No & $118(53,9 \%)$ \\
& Ninguna & $73(33,0 \%$ \\
& Hipertensión & $73(33,0 \%)$ \\
& Diabetes & $38(18 \%)$ \\
& Pulmonar & $65(29,4 \%)$ \\
Ingresos previos & Cardíaca & $19(8,6 \%)$ \\
& Psiquiátrica & $16(7,2 \%)$ \\
Estancia media & Otros & $45(20,4 \%)$ \\
& 0 & $134(61,2 \%)$ \\
& $1-2$ & $72(32,9 \%)$ \\
& $>2$ & $13(5,9 \%)$ \\
\hline & 8,7 días & \\
\hline \multirow{2}{*}{ Consumo acumulado } & 43 paquetes/año \\
\hline
\end{tabular}

El diagnóstico al alta más frecuente era neumonía $(72,32,9 \%)$, seguido de neoplasias en 47 sujetos $(21,5 \%)$ y $42(19,2 \%)$ de EPOC o exacerbación de EPOC. Otros diagnósticos al alta fueron: neumotórax, derrame pleural, crisis de asma, tromboembolismo pulmonar, tuberculosis e hipertensión pulmonar (Tabla 2). La estancia media fue de 8,7 días. De los que han dejado de fumar el diagnóstico al alta más frecuente fue neumonía $(29,6 \%)$ seguido de EPOC o agudización de EPOC $(20,4 \%)$ y neumotórax $(5,6 \%)$ (Figura 1).

En cuanto al hábito la media medida en índice paquetes/años era de 42. En sus historias podía recogerse la intervención que habían tenido durante su ingreso, a 45 de ellos $(20,6 \%)$ se le impartió consejo médico, a 2 se les efectuó tratamiento farmacológico $(0,9 \%)$ y sobre 172 $(78,5 \%)$ no se realizó ninguna intervención o no quedaron registradas en sus informes al alta (Figura 2).

Tras un año posterior al alta, y ponernos en contacto con los pacientes, 94 (42,9\%) continúan fumando, reconociendo que durante una media de 49,3 días (el 50\%) dejaron de fumar. En cambio $67(30,6 \%)$ sujetos, han abandonado el tabaco tras el ingreso en el hospital. Se perdieron 58 sujetos $(26,5 \%)$ con los que no se pudo contactar. De los que han dejado de fumar el $75,9 \%$ no recibieron ningún tipo de intervención, el 22,2\% recibieron consejo médico y un $1,85 \%$ tratamiento farmacológico. El abandono del tabaco en los pacientes que decían haber dejado de fumar quedó reflejado en COoximetría que venían a realizar en el hospital con una media de 4,2 ppm $( \pm 2,7$ DS) lo que confirma que la tasa de abstinencia era real.

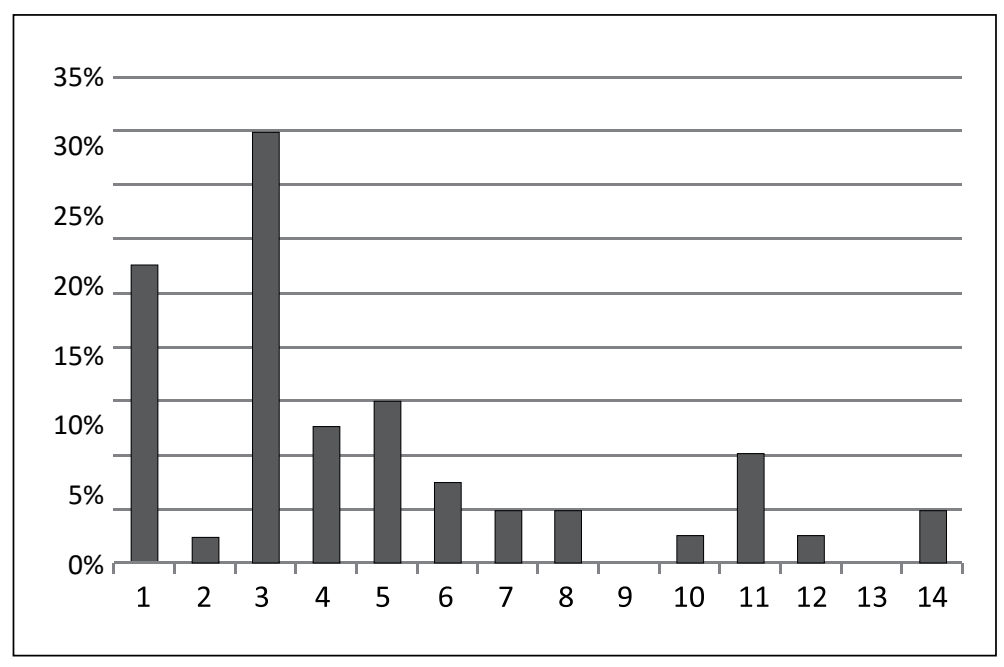

Figura 1. Diagnóstico al alta de los sujetos que han dejado de fumar. (1. EPOC o Agudización de EPOC, 2. Crisis asmática o asma; 3. Neumonía; 4. Neoplasia; 5. Neumotórax; 6. Tromboembolismo pulmonar; 7. Derrame pleural; 8. Tuberculosis; 9. Bronquiectasias; 10. Casi ahogamiento; 11. Insuficiencia respiratoria; 12. Síndrome de Apnea Obstructiva del sueño: SAOS; 13. Hipertensión pulmonar; 14. otros). 
Intervención recibida

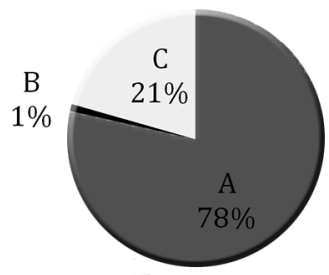

Tabaquismo al alta

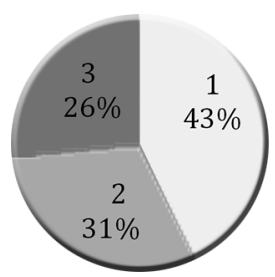

Figura 2. Intervención médica realizada: A) Ninguna; B) Tratamiento farmacológico; C) Consejo médico. Tabaquismo al alta: 1) Continúan fumando; 2) No fuman; 3) Pérdidas.

\section{Comentarios}

El ingreso hospitalario ofrece al fumador una oportunidad ideal para abandonar el tabaco, viéndose esto favorecido por diversas circunstancias. El paciente es consciente, y debemos exponérselo, de la relación directa del tabaco con la patología por la que ha ingresado para que perciba la gravedad del hábito tabáquico, además, el hospital es un espacio libre de humo donde estará protegido de factores externos que lo precipiten a fumar. Todo ello, junto al contacto directo con profesional sanitario crea un marco idóneo para comenzar la deshabituación tabáquica. El tabaquismo es reconocido por la Organización Mundial de la Salud (OMS) entre otras entidades, como una enfermedad crónica y se trata además de una sustancia adictiva $^{8}$, así todos los pacientes ingresados en un hospital deben ser interrogados sobre su hábito tabáquico, tal es así que algunos autores postulan que debe ser registrado como otra constante vital ${ }^{9}$. Ello cobra especial importancia en el Servicio de Neumología, por tratarse de una causa directa de enfermedades respiratorias. Una enfermedad tan prevalente como la enfermedad pulmonar obstructiva crónica (EPOC) es originada en un 85 a $90 \%$ de los casos, por el consumo del tabaco ${ }^{10}$ y hasta el 20\% de pacientes con EPOC que están ingresados en los hospitales españoles consumen tabaco ${ }^{11}$. Estos sujetos tienen además una alta dependencia a la nicotina y una baja motivación para abandonar el tabaco por lo que la ayuda del especialista médico cobra mayor relevancia ${ }^{12,13}$.

El estudio de la cesación tabáquica tras un ingreso hospitalario en el Servicio de Cardiología ha sido objetivo de múltiples estudios ${ }^{14-16}$ en cambio, ¿Qué sucede cuando el fumador es dado de alta del servicio de Neumología? Desconocemos el impacto que supone el propio ingreso en sí y lo que ocurre con esos sujetos una vez que abandonan el centro. Los datos recogidos muestran una mayor frecuencia en los hombres que en las mujeres, con una media de edad de 56 años. Los costos derivados del tratamiento médico de enfermedades crónicas, del propio ingreso, sumado a la ausencia del puesto de trabajo justificaría políticas con programas que fomenten la cesación tabáquica ya que queda demostrado que son costo efectivas ${ }^{17-19}$.

$\mathrm{Al}$ ingreso, la mayoría de ellos tienen alguna enfermedad diagnosticada previamente, lo cual demostraría la relación del tabaco no sólo con enfermedades respiratorias sino con otro tipo de patologías.

Si nos fijamos en nuestros pacientes, hubo un mayor abandono entre los sujetos que ingresaban por primera vez en el hospital. El impacto de requerir una primera hospitalización debe ser considerado el momento óptimo para impulsar al sujeto a dejar de fumar ya que probablemente esté más susceptible, sin embargo, ante individuos con ingresos repetidos en diferentes servicios debemos considerar que tendrán alta dependencia requiriendo otras estrategias de ayuda, que incluyan un mayor seguimiento y apoyo, interrogando si ha tenido intentos previos para dejar de fumar que nos permitan analizar cuál es la mejor manera de abordar su cesación tabáquica.

Los estudios que han comparado las cifras de abstinencia diferenciando por servicios médicos han obtenido cifras mayores para los ingresados por patología cardíaca seguidos de los enfermos pulmonares ${ }^{20}$. Los pacientes que con mayor frecuencia reconocieron haber dejado de fumar fueron los que su diagnóstico al alta es de neumonía seguido del diagnóstico de EPOC. Patologías más graves como el diagnóstico de una neoplasia ocupa una cuarta posición. No se relaciona la gravedad de la enfermedad con la mayor motivación para dejar de fumar, por lo que no se debe creer que haber diagnosticado una enfermedad de la gravedad de un cáncer, hará por sí mismo que el sujeto deje de fumar.

Los síntomas del síndrome de abstinencia aparecen aproximadamente a las 4 o $12 \mathrm{~h}$ de la cesación del tabaco ${ }^{21}$, durando de una a varias semanas, por lo que con una estancia media de 
nuestros pacientes de 8,7 días podemos presuponer que estos síntomas iniciales consecuencia de dejar de fumar, los padecería el sujeto en nuestro servicio, por lo que habría que detectarlo para poder apoyar al paciente y ofrecerle tratamiento, pues se sabe que es un factor predictor en el fracaso del abandono del tabaco ${ }^{22}$. Si estos síntomas son reconocidos por el fumador y aliviados mientras está rodeado de personal sanitario estará preparado para afrontarlos una vez que esté expuesto a su entorno.

Los ingresados tenían un grado de tabaquismo grave (media de 43 paquetes/año) por lo que se trata de sujetos de riesgo y de alta complejidad para dejar de fumar, a pesar de eso en los informes de alta de 172 sujetos no queda registrado que el profesional médico especialista en neumología realizara ninguna intervención, de ahí la necesidad del entrenamiento del profesional médico, que está aún lejos de lo deseable en materia de formación en el manejo de tabaquismo. No se puede discutir la necesidad de preguntar al paciente que si es fumador ${ }^{23,24}$ ya que una intervención conductual o farmacológica aumenta la cesación tabáquica $^{25}$. Como profesionales sanitarios, al ser un problema de salud pública y una enfermedad crónica, debe ser siempre abordado ofreciendo un tratamiento, al igual que tratamos otras patologías, el tabaco es una más y no es concebible que no se les ayude a los fumadores para dejar de serlo. Diferentes guías ${ }^{26,27}$ dan las directrices al personal sanitario de cómo atender a las poblaciones especiales como jóvenes y embarazadas a la vez que muestran cómo usar las diferentes terapias farmacológicas y dan respuesta a los problemas más frecuentes que hay que abordar en nuestros pacientes.

Nos pusimos en contacto con nuestros pacientes transcurrido al menos un año desde el alta, para preguntarle si habían dejado de fumar suponiendo que la intervención en sí ha sido el propio ingreso hospitalario. La tasa de abstinencia al mes es del $52 \%$ siendo esta cifra superior al compararla con otros estudios, como el del Dr. Roig Cutillas ${ }^{20}$ que obtiene una cesación tabáquica del $35,6 \%$. Al año alcanzamos una tasa del 30,6\%. Sin embargo, un estudio sobre enfermos cardiovasculares con una intervención mínima alcanzan hasta una abstinencia del $62,2 \%$ a los 12 meses $^{28}$. En otros estudios en pacientes cardiovasculares en los que realizan intervenciones, obtienen a los seis meses abstinencias del $67 \%$ y del $49 \%$ con diferencias significativas comparándolos con los grupos que sólo reciben un cuidado mínimo donde las cifras caen hasta el $43 \%$ en el trabajo de Dornelas ${ }^{29}$. Por lo que si en nuestra población realizáramos alguna intervención esperaríamos encontrar cifras superiores. Entre los sujetos que han dejado de fumar solo una quinta parte recibió consejo médico por lo que confirma que el propio ingreso en sí supuso un impacto para abandonar el tabaco. Se confirmó la fiabilidad de las respuestas de los pacientes que afirmaban haber dejado de fumar al obtener una media de 4,2 ppm en la COoximetría. Si bien se sabe que las respuestas son fiables ${ }^{30}$, la propia COoximetría pudo servir de factor motivador para permanecer sin fumar. Otro dato a tener en cuenta es que la mitad de los que continúan fumando estuvieron una media de 49 días sin fumar, por lo que si en este tiempo hubieran recibido una intervención nuestras cifras de abstinencia serían mayores ya que el contacto posterior aumenta las cifras.

\section{Conclusión}

El propio ingreso hospitalario ofrece en sí un marco ideal para abandonar el tabaco. La presencia del personal sanitario para motivar y aliviar los síntomas derivados de la abstinencia junto al contexto de un espacio libre de humo es una oportunidad que el profesional médico debe reconocer. Es necesario formar a los especialistas para diagnosticar y tratar al paciente en dicho proceso, de tal forma que sobre todo sujeto fumador hospitalizado se realice alguna intervención. Todos los centros deben tener consultas monográficas donde se pueda realizar un seguimiento que permita aumentar las cifras de abstinencia.

\section{Bibliografía}

1.- BANEGAS J R, DIEZ-GAÑÁN L, BAÑUELOS-MARCO B, GONZÁLEZ-ENRÍQUEZ J, VILLAR- ÁLVAREZ F, MARTÍN-MORENO J M, et al. Mortalidad atribuible al consumo de tabaco en España en 2006. Medicina Clínica (Barc) 2011; 136: 97-102.

2.- SASCO A J, SECRETAN M B, STRAIF K. Tobacco smoking and cancer: a brief review of recent epidemiological evidence. Lung Cancer 2004; 45 Suppl 2: 3-9.

3.- TEO K K, OUNPUU S, HAWKEN S, PANDEY M R, VALENTIN V, HUNT D, et al. Tobacco use and risk of myocardial infarction in 52 countries in the Interheart study: a case-control study. Lancet 2006; 368: 647-58.

4.- RIESCO MIRANDA J A. Arch Bronconeumol. Efectos "no respiratorios" del tabaco. 2007; 43: 477-8. DOI: $10.1157 / 13109466$.

5.- RIGOTTI N A, CLAIR C, MUNAFO M R, STEAD L F. Cochrane Database Syst Rev. 2012 May 16; 5: CD001837. doi: 10.1002/14651858.CD001837.pub3. Interventions for smoking cessation in hospitalized 
patients.

6.- SCIAMANNA C N, STILLMAN F A, HOCH J S, BUTLER J H, GASS K G, FORD. Opportunities for improving inpatient smoking cessation programs: a community hospital experience. Prev Med 2000; 30: 496-503.

7.- RIGOTTI N A, MUNAFO M R, STEAD L F. Intereventions for smoking cessation in hospitalized patients (Cochrane Review). En: The Cochrane Library 2008, Issue 4. Chichester, RU: Wiley and Sons, Ltd.

8.- OTERO M, AYESTA F J. El tabaquismo como trastorno adictivo. Elsevier.

9.- FIORE M C. The new vital sign. Assessing and documenting smoking status. JAMA 1991; 266: 3183-4.

10.- Global Strategy for the Diagnosis, Management and Prevention of COPD, Global Initiative for Chronic Obstructive Lung Disease (GOLD) 2011 [consultado el 01 de enero de 2012]. Disponible en: http://www.goldcopd.org/.

11.- POZO F, ÁlVAREZ C J, CASTRO A, MELERO C, CAPELASTEGUi A, ESTEBAN C, et al. Auditoría clínica de los pacientes hospitalizados por exacerbación de EPOC en España (estudio AUDIPOC): Método y organización del trabajo. Arch Bronconeumol 2010; 46: 349-57.

12.- SHAHAB L, JARVIS M J, BRITTON J, WEST R. Prevalence, diagnosis and relation to tobacco dependence of chronic obstructive pulmonary disease in a nationally representative population sample. Thorax 2006; 61: 1043-7. doi: 10.1136/thx.2006.064410.

13.- JIMÉNEZ-RUIZ C A, RIESCO MIRANDA J A, ALTET GÓMEZ C N, LORZA BLASCOD J J, SIGNES-COSTA J, SOLANO REINAF S, et al. Tratamiento del tabaquismo en fumadores con enfermedad pulmonar obstructiva crónica. Normativa SEPAR. Arch Bronconeumol 2013; 49: 354-63.

14.- MÍGUEZ M C, PEREIRA B. Intervenciones para dejar de fumar con pacientes hospitalizados por enfermedad cardiovascular: una revisión de la literatura. Universidad de Santiago de Compostela. Health and Addictions 2013; 13: 123-34.

15.- SIVARAJAN E S, MILLER N H, CHRISTOPHERSON D J, MARTIN K, PARKER K M, AMONETI M, et al. High rates of sustained smoking cessation in women hospitalized with cardiovascular disease the women's initiative for nonsmoking (WINS). Circulation 2004; 109: 587-93.

16.- DORNELAS E, SAMPSON R, GRAY J, WATERS D, THOMPSON P. A randomized controlled trial of smoking cessation counselling after myocardial infarction. Preventive Medicine 2000; 30: 261-8.

17.- SONG F, RAFTERY J, AVEYARD J, HYDE C, BARTON P, WOOLACOTT N. Cost-effectiveness of pharmacological interventions for smoking cessation: a literature review and decision analytic analysis. Med Decis Making 2002; 22 suppl S26-S37.

18.- RICHARD P, WEST K, KU L. The return on investment of a Medicaid tobacco cessation program in
Massachusetts. PLoS ONE 2012; 7: e29665.

19.- TAYLOR M, LEONARDI-BEE J, AGBOOLA S, MCNEILL A, COLEMAN T. Cost effectiveness of interventions to reduce relapse to smoking following smoking cessation. Addiction 2011; 106: 1819-26.

20.- ROIG CUTILLAS P, SABATER PUIG E, BORRÀS CABACÉS T, SESMILO GARCÍA M, SALMONS ALEU R, ALBELDO NAVARRO V. Protocolo de intervención en pacientes fumadores hospitalizados. Prev Tab 2001; 3: 124-31.

21.- SHIFFMAN S, WEST R J, GILBERT D G. SRNT Work Group on the Assessment of Craving and Withdrawal in Clinical trials. Nicotine Tob Res 2004; 6 : 599-614.

22.- PIÑEIRO B, LÓPEZ-DURÁN A, FERNÁNDEZ DEL RÍO E, MARTÍNEZ U, BRANDON T. BECOÑA E. Craving and nicotine withdrawal in a Spanish smoking cessation simple. Adicciones 2014; 26: 230-7.

23.- RAW M, MCNEILL A, WEST R J. Smoking cessation guidelines for health care professionals. Thorax 1998; 53 (Suppl 5, Part 1): S1-S19.

24.- WEST R, MCNEILL A, RAW M. Smoking cessation guidelines for health professionals: an update. Thorax 2000; 55: 987-99.

25.- PATNODE C D, HENDERSON J T, THOMPSON J H, SENGER C A, FORTMANN S P, WHITLOCK E $P$. Behavioral Counseling and Pharmacotherapy Interventions for Tobacco Cessation in Adults, Including Pregnant Women: A Review of Reviews for the U.S. Preventive Services Task Force. Ann Intern Med 2015; 163: 608-21.

26.- ANDERSON J E, JORENBY D E, SCOTT W J, FIORE M C. Treating tobacco use and dependence: an evidence-based clinical practice guideline for tobacco cessation. Chest 2002; 121: 932-41.

27.- MINISTRY OF HEALTH. 2007. New Zealand Smoking Cessation Guidelines. Wellington: Ministry of Health.

28.- MOCHÓN RAMOS S. Breve intervención de cesación tabáquica en enfermos cardiovasculares hospitalizados. Revista Española de Cardiología 2009; 62: 447-50.

29.- DORNELAS E, SAMPSON R, GRAY J, WATERS D. THOMPSON P. A randomized controlled trial of smoking cessation counselling after myocardial infarction. Preventive Medicine 2000; 30: 261-8.

30.- BARRUECO FERRERO M, JIMÉNEZ RUIZ C A, PALOMO A, TORECILLA M, ROMERO P J, RIESCO J A. Veracidad de las respuestas de los fumadores en las consultas de deshabituación tabáquica sobre su abstinencia. Arch Bronconeumol 2005; 41: 135-40.

Correspondencia a:

Dra. Eva Cabrera Cesar

Servicio de Neumología, Hospital Universitario

Virgen de la Victoria. Málaga, España.

Email: evacabreracesar@gmail.com 